New therapies

\title{
The potential role of soluble fibre in the treatment of hypercholesterolaemia
}

\author{
Andrew J S Coats
}

\begin{abstract}
Summary
The three major modifiable coronary risk factors are smoking, hypertension, and hypercholesterolaemia. Serum cholesterol levels are above the desirable level of $5.2 \mathrm{mmol} / \mathrm{l}$ in $79 \%$ of men and $65 \%$ of women aged between 35 and 50 years and thus are an important target for intervention. In this paper, the role of nonpharmacological intervention with soluble fibre in treating mild to moderate primary hypercholesterolaemia is reviewed. Evidence from controlled studies shows that soluble fibre can be effective in lowering cholesterol by clinically significant amounts. It is stressed, however, that risk factors for coronary heart disease are interactive and attention is shifting to addressing multiple rather that individual factors.
\end{abstract}

Keywords: fibre; cholesterol; ischaemic heart disease

Imperial College of Science, Technology and Medicine at the National Heart and Lung Institute; and Department of Cardiology, Royal Brompton Hospital, London SW3 6NP, UK

A J S Coats

Accepted 21 January 1998
Coronary heart disease (CHD) is the leading cause of death in men over the age of 45 years and women over the age of 65 years in Europe. ${ }^{1}$ As a result, attempts to reduce this burden of disease are of paramount importance, and a reduction in the occurrence and progression of CHD remain major goals of preventive medicine. The UK Government's Health of the nation document sets a target for reducing mortality from CHD in people under 65 years of age by at least $40 \%$ by the year $2000 .^{2}$

Of the three major modifiable coronary risk factors, smoking, hypertension, and hypercholesterolaemia, the last is the one most relevant to the population as a whole. Serum cholesterol levels are above the desirable level of $5.2 \mathrm{mmol} / \mathrm{l}$ in $79 \%$ of men and $65 \%$ of women aged between 35 and 50 years. ${ }^{3}$ We must differentiate between primary and secondary prevention, and between those with genetically determined grossly elevated cholesterol levels who almost always require drug therapy, and those with more mildly elevated cholesterol levels due to acquired dietary factors where dietary modifications may be sufficient. Most would agree there is an important place for cholesterol reduction in secondary prevention regardless of age, although controversy still exists on the role of cholesterol reduction for primary prevention in those with no evidence of vascular disease over the age of 60 years. Practice guidelines of how to manage hypercholesterolaemia to reduce CHD have been produced by both the British Hyperlipidaemia Association ${ }^{4}$ and a European Society of Cardiology Task Force, ${ }^{1}$ with broadly similar recommendations. Newer revised guidelines from the European Atherosclerosis Society are awaited. Aside from lifestyle and dietary interventions, effective pharmacological treatments for hypercholesterolaemia have been developed, especially the hydroxymethylglutaryl coenzyme A reductase inhibitors (the 'statins'). The cost of these agents is, however, high, and as the majority of the burden of CHD falls on people with only mildly to moderately elevated cholesterol levels, interventions which are economically affordable and widely applicable are needed to maximise prevention of CHD. Patients are frequently resistant to taking medication unless symptomatic, and as many as $50 \%$ of prescriptions for chronic asymptomatic risk factors such as hypertension are not continued after 6 months. It is likely that a similar phenomenon may affect hypocholesterolaemic prescriptions. It is also likely that a larger population could be reached by 'non-pharmaceutical' remedies for risk factors in which the general population may have a greater confidence regarding safety.

This paper reviews the evidence that high cholesterol levels are linked to the rates of morbidity and mortality from CHD, and the strategies available to reduce these risks. It specifically addresses the issue of whether there is a role for non-pharmacological intervention with soluble fibre in treating mild to moderate primary hypercholesterolaemia. It must be borne in mind that risk factors for CHD are interactive and attention is shifting to addressing multiple risk factors rather that individual factors in isolation.

\section{Evidence that raised cholesterol increases CHD mortality}

Many population studies have shown that the risk of mortality from CHD increases with rising average levels of plasma cholesterol. ${ }^{45}$ In the Framingham study - a prospective cohort study - an elevated blood cholesterol was associated with an increased risk of future coronary heart disease. ${ }^{6}$ Other cohort studies have confirmed this finding. ${ }^{78}$ The Multiple Risk Factor Intervention Trial (MRFIT) screened a third of a million men aged between 35 and 57 years and followed most of these men for 6 years. The higher an individual's cholesterol level, the greater was his risk of death from CHD. ${ }^{10}$ 


\section{Causes of hypercholesterolaemia}

Inherited genetic disorders explain a small percentage of cases of hypercholesterolaemia. The most common are familial combined hyperlipidaemia, familial hypercholesterolaemia, and familial hypertriglyceridaemia. ${ }^{11}$ Data from the US suggest that these conditions affect between 0.2 and $0.5 \%$ of the population. More people than this have a genetic disposition to hyperlipidaemia, but the genetic basis of this predisposition is not known. ${ }^{1}$

In the majority of cases with only mild to moderate hypercholesterolaemia, the cause is primarily dietary. Dietary saturated fats raise plasma cholesterol and potentiate the hypercholesterolaemic effect of dietary cholesterol. ${ }^{4}$ Other lifestyle factors play a role in determining the eventual cholesterol level for an individual. A high level of habitual physical activity is associated with lower levels of plasma LDL-cholesterol and triglycerides, and higher levels of the protective HDL-cholesterol. ${ }^{1}$ Drug therapy may also play a role in determining cholesterol levels. Inevitably many patients with angina or after a myocardial infarction will be started on a $\beta$-blocker, despite their adverse impact on the lipoprotein profile, particularly VLDL-cholesterol.

High cholesterol levels can occasionally occur secondary to other disorders, such as hypothyroidism, diabetes mellitus, and in heavy alcohol imbibers. Lipid levels can also be increased by certain drugs (frequently used in patients at increased risk, such as hypertensives). These include $\beta$-blockers, thiazide diuretics, and oral contraceptive agents. ${ }^{11}$ Coronary risk factors may co-exist more frequently than would be expected by chance, such as the association between hypertension, hyperlipidaemia, central obesity, and insulin resistance or even frank diabetes. The drugs used to correct one risk factor (hypertension) may worsen another (hypercholesterolaemia) so that the net effect of treatment in terms of CHD prevention may be less than expected on the basis of correcting isolated risk factors. Some pairs of risk factors are particularly important in combination. For example, there is some suggestion that smoking may be far less of a risk factor for CHD in the absence of elevated LDL-cholesterol levels. ${ }^{1}$ This might explain why smoking does not cause more CHD in southern European countries. It is increasingly realised, however, that it is the percentage reduction in lipoprotein levels that matters in those at risk of CHD, rather that the absolute lipoprotein levels.

\section{Effect of reducing raised cholesterol on CHD mortality}

\section{PRIMARY PREVENTION}

In the West of Scotland Coronary Prevention Study (WOSCOPS), ${ }^{12}$ men at high risk of CHD, but not diagnosed as having established CHD, aged 45 to 64 years and with a plasma cholesterol level above $6.5 \mathrm{mmol} / 1$ were advised to modify their diet in order to lower cholesterol. Those with persistently raised cholesterol were randomised to receive either pravastatin or placebo. A total of 6595 men were treated and followed for an average of 4.9 years. Total cholesterol was reduced by $20 \%$ in the active treatment group. This group suffered 174 heart attacks during follow-up compared with 248 heart attacks in the placebo group, a $31 \%$ risk reduction, which was statistically significant. Active cholesterol-lowering reduced both fatal and non-fatal myocardial infarctions.

\section{SECONDARY PREVENTION}

In the Scandinavian Simvastatin Survival Study (4S), ${ }^{13} 4444$ patients with angina or a past history of myocardial infarction were randomised to receive either simvastatin or placebo if their serum cholesterol levels were in the range 5.5 to $8.0 \mathrm{mmol} / 1$ after dietary advice. Over a follow-up period averaging 5.4 years, in the group receiving active therapy, total cholesterol was $25 \%$ lower and the death rate from CHD was $42 \%$ lower $(p<0.0003)$ than in the placebo group.

The Cholesterol and Recurrent Events (CARE) trial ${ }^{14}$ was designed specifically to investigate the effectiveness of reducing LDL-cholesterol levels to below the average level of $4.5 \mathrm{mmol} / 1$ to prevent recurrent coronary artery events in a post-myocardial infarction population. A total of 4159 men and women aged 21 to 75 years (with average baseline total cholesterol of $5.4 \mathrm{mmol} / \mathrm{l}$ ) were randomised to receive either pravastatin or placebo and were followed for five years. Active cholesterol-lowering therapy significantly reduced non-fatal myocardial infarction and CHD death by $24 \%$. The majority of the patients in these studies were male; with the exception of the CARE study, there are very few data on women. 
RESULTS OF META-ANALYSES

A meta-analysis of the large prospective cohort studies, international comparisons, and randomised controlled trials of cholesterol lowering published in 1994 concluded that a difference in total cholesterol of $0.6 \mathrm{mmol} / 1$ was associated with a $27 \%$ decrease in risk of CHD mortality. ${ }^{15}$ Further estimates are that in mild to moderate hypercholesterolaemia, a $10 \%$ reduction in total cholesterol would reduce CHD risk by $50 \%$ in a 40 -year-old, $40 \%$ in a 50 -year-old, $30 \%$ in a 60 -year-old, and by $20 \%$ in a 70 -year-old. ${ }^{16}$ Given the higher prevalence of CHD and greater risk of fatal CHD in the elderly, these percentage reductions are more likely to lead to substantial increases in disease-free survival in the elderly, despite the smaller percentage reductions in CHD risk.

\section{Cholesterol-lowering strategies}

\section{DIETARY METHODS}

The guidelines of the British Hyperlipidaemia Association and the European Atherosclerosis Society recommend that dietary measures and lifestyle measures should be tried before considering pharmacological lipid-lowering therapies. ${ }^{14}$ The aim of dietary modification is to reduce fat intake so that no more than $30 \%$ of total dietary calories are fat-derived. ${ }^{4}$ Saturated fat should be restricted to less than $10 \%$ of total calorie intake, especially as saturated fat intake raises plasma cholesterol levels and increases the hypercholesterolaemic effect of dietary cholesterol. In addition, cholesterol intake should be restricted to $300 \mathrm{mg} /$ day for an adult, and part of the diet replaced by complex carbohydrate, particularly soluble fibre. What these dietary targets mean in terms of usual diet is beyond the scope of this article, but useful diet fact sheets are available from dieticians.

In practice, dietary modifications have proved disappointing. Long-term clinical studies have shown a reduction in cholesterol achieved through voluntary dietary modification to be no more than $2 \% .{ }^{17-20}$

\section{DRUG THERAPY}

Several drug classes are available to clinical practice to reduce cholesterol levels. These include anion-exchange resins (cholestyramine, colestipol), probucol, fibric acid derivatives (clofibrate, bezafibrate, ciprofibrate, fenofibrate, gemfibrozil), nicotinic acid and its analogues, and statins (pravastatin, simvastatin, etc). These agents, especially the statins, give substantial (up to $25 \%$ ) reductions in cholesterol levels. They are extremely effective at reducing cholesterol levels and have been the drugs used in the very effective primary and secondary prevention trials reviewed above. The choice of which drug to use should take into consideration the nature of the underlying lipid metabolism abnormality. Simvastatin and pravastatin, in addition to their proven role in secondary prevention of $\mathrm{CHD}$, are also first-line treatments for individuals who have a pure or combined hypercholesterolaemia, excluding children and women of child-bearing potential. The anticipated benefit of lipid-lowering medication must be weighed, however, against the cost and patient resistance to long-term drug therapy, as well as the possibility and fear of side-effects. ${ }^{4}$ The fear of side-effects is probably exaggerated; reports of rhabdomyolysis and very high creatine kinase levels have only been isolated cases and the incidence of side-effects in the large randomised trials has been very low. Nevertheless, fear of (perceived) side-effects remains a major reason for poor patient compliance.

\section{SOLUBLE FIBRE SUPPLEMENTS}

Soluble fibre in various forms, particularly ispaghula husk or psyllium, has been shown to reduce serum lipid levels. ${ }^{21}$ It has very interesting properties and there is considerable interest in its potential. It has been used for some time in the treatment of irritable bowel syndrome and Dr Alan Howard of the Dunn Nutrition Unit in Cambridge was using psyllium in the early 1970s as a lipid-lowering agent. In placebo-controlled trials, increasing dietary fibre has reduced cholesterol levels by up to $15 \% .^{22-27}$ There are a number of theories to explain how soluble fibre may reduce serum cholesterol $^{21}$ :

- the viscous nature of soluble fibre may decrease absorption of cholesterol from the gut

- soluble fibre may increase excretion of bile acids in the faeces. To compensate the liver produces more bile acids from cholesterol

- fermentation of soluble fibre by colon bacteria producing short-chain fatty acids, including proprionates. Proprionate is absorbed and converted to succinyl-coenzyme A in the liver where it may inhibit cholesterol synthesis. 
IS THERE A ROLE FOR SOLUBLE FIBRE IN THE TREATMENT OF CHD?

There is abundant evidence that hypercholesterolaemia contributes to the risk of premature $\mathrm{CHD}$, and that lowering cholesterol levels decreases this risk. Substantial reductions in mortality and CHD events have been achieved by the statin group of drugs, most notably in secondary prevention or high-risk individuals. The difficulty, however, is in deciding how or whether to treat mild or moderate primary hypercholesterolaemia in other groups (who constitute the bulk of the population who will eventually suffer CHD events). However attractive in theory, dietary modification has not proved as effective in practice as could have been hoped. This may be due to poor compliance with dietary advice or poor selection of patients. Those with genetically determined hypercholesterolaemia or who have diabetes are less likely to respond adequately to dietary manipulation alone, for they have a particular metabolic abnormality that requires specific therapeutic intervention. Lipid-lowering drugs are expensive, not without side-effects, and remain recommended only in secondary prevention or high-risk individuals. More strategies for primary prevention in the larger population remain, therefore, necessary.

Soluble fibre can be effective in lowering cholesterol by clinically significant amounts, and is essentially non-pharmacological (although a small amount of proprionate derived from fibre may be absorbed). Soluble fibre-based products containing ispaghula husk could provide an appropriate long-term maintenance treatment in conjunction with dietary modification for those patients with mild or moderately elevated serum cholesterol whose risk of $\mathrm{CHD}$, although increased, does not merit the use of systemic hypolipidaemic drugs. Thus, a convenient form of soluble fibre which is well tolerated, cost-effective, and has been shown to reduce cholesterol levels in placebo-controlled clinical trials would be a valuable addition to the clinician's armamentarium.

This work was supported by the Viscount Royston Trust and the British Heart Foundation.

1 Pyörälä K, De Backer G, Graham I, PooleWilson P, Wood D. Prevention of coronary heart disease in clinical practice: recommendations of the Task Force of the European Society of Car-
diology, European Atherosclerosis Society and European Society of Hypertension. AtheroscleroEuropean Society of Hyp
sis 1994;110:121-61.

2 The health of the nation - a strategy for health in England. Government White Paper, July 1992 3 Gregory J, Foster K, Tyler H, Wiseman M. Th dietary and nutritional survey of British adults: a survey of the dietary behaviour, nutritional status and blood pressure of adults aged 16-24. London HMSO, 1990.

4 Betteridge DJ, Dodson PM, Durrington PN, et al. Management of hyperlipidaemia: guideline of the British Hyperlipidaemia Association. Postgrad Med ₹ 1993;69:359-69.

5 Simons LA. Interrelations of lipids and lipoproteins with coronary artery disease mortality in

19 countries. Am f Cardiol, 1986;57:5G-10G.

Kannel WB, Gordon T, Castelli WP. Role of the Framingham Study. Progr Lipid Res 1981;20: the Framing.

7 Goldbourt V, Holzman E, Newfeld HN. Total and high density lipoprotein cholesterol in the serum and risk of mortality: evidence of a threshold effect. $B M \mathcal{F}$ 1985;290:1239-43.

8 The Pooling Project Research Group. Relationship of blood pressure, serum cholesterol, smoking habit, relative weight and ECG abnormalities to incidence of major coronary events. $\mathcal{f}$ Chron Dis 1978;31:201-6.

9 Martin MJ, Hulley SB, Browner WS, Kuller LH, Wentworth D. Serum cholesterol, blood pressure and mortality: implications from cohort of 361,662 men. Lancet 1986;2:933-6.

10 Stamler J, Wentworth $D$, Neaton JD. Is relationship between serum cholesterol and risk of premature death from coronary heart disease continuous and graded? Findings in 356,222 primary screenees of the Multiple Risk Factor Intervention Trial (MRFIT). $\mathscr{f} A M A$ 1986;256: 2823-8.
11 Bilheimer DW. Disorders of lipid metabolism. In: Kelly W, ed. Textbook of internal medicine. Philadelphia: JB Lipincott Company, 1992; pp2052-5.

12 Shepherd J, Cobbe SM, Ford I, et al. Prevention of coronary heart disease with pravastatin in men with hypercholesterolaemia. $N \mathrm{Engl} \mathrm{f} \mathrm{Med}$ 1995;333:1301-7.

13 Scandinavian Simvastatin Survival Study Group. Randomised trial of cholesterol lowering in 4444 patients with coronary heart disease: the Scandinavian Simvastatin Survival Study (4S). Lancet 1994;344:1383-9.

14 Sacks FM, Pfeffer MA, Moye LA, et al. The effect of pravastatin on coronary events after myocardial infarction in patients with average myocardial infarction in patients with average
cholesterol levels. $N$ Engl $¥$ Med 1996;335:10019.

15 Law MR, Wald NJ, Thompson SG. By how much and how quickly does reduction in serum cholesterol concentration lower risk of ischaemic heart disease? [see comments]. BMF 1994 308:367-72.

16 Betteridge DJ. Cholesterol lowering and CHD prevention: time to get on with it. Br f Clin Pract 1994;48:115-7.

17 Ramsay L, Yeo WW, Jackson PR. Dietary reduction of serum cholesterol concentration: time to think again [see comments]. BMf 1991; time to think

18 Family Heart Study Group. Randomised controlled trial evaluating cardiovascular screening and intervention in general practice: principa results of British family heart study. $B M \mathcal{F} 1994$; 308:313-20.

19 Neil HAW, Roe L, Godlee RJ, et al. Randomised trial of lipid lowering dietary advice in general practice: the effects of serum lipids, lipopro-
teins, and antioxidants. $B M \mathcal{F} 1995 ; 310: 569-73$.

20 Watts GF, Lewis B, Brunt JN, et al. Effects on coronary artery disease of lipid-lowering diet, or diet plus cholestyramine, in the St Thomas Atherosclerosis Regression Study (STARS) [see comments]. Lancet 1992;339:563-9.
21 Glore SR, van Treeck D, Knehans AW, Guild $M$. Soluble fiber and serum lipids: a literature review. $\mathcal{f}$ Am Diet Assoc 1994;94:425-36.

22 Anderson JW, Zettwoch N, Feldman T, Tietyen Clark J, Oeltgen P, Bishop CW. Cholesterollowering effects of psyllium hydrophilic mucilloid for hypercholesterolemic men. Arch Intern Med 1988;148:292-6.

23 Bell LP, Hectorn K, Reynolds H, Balm TK, Hunninghake DB. Cholesterol-lowering effects of psyllium hydrophilic mucilloid. Adjunct therapy to a prudent diet for patients with mild to moderate hypercholesterolaemia. $\mathfrak{F A M}$ 1989;261:3419-23.

24 Levin EG, Miller VT, Muesing RA, Stoy DB Balm TK, LaRosa JC. Comparison of psyllium hydrophilic mucilloid and cellulose as adjuncts to a prudent diet in the treatment of mild to moderate hypercholesterolemia. Arch Intern Med 1990;150:1822-7.

25 Anderson JW, Floore TL, Geil PB, O’Neal DS Balm TK. Hypocholesterolemic effects of different bulk-forming hydrophilic fibers as adjuncts to dietary therapy in mild to moderate hypercholesterolemia. Arch Intern Med 1991, 151:1597-602.

26 Bell LP, Hectorn K, Reynolds H, Hunninghake DB. Cholesterol-lowering effects of solublefiber cereals as part of a prudent diet for patients with mild to moderate hypercholesterolaemia. Am 7 Clin Nutr 1990;52:1020-6.

27 Haskell WL, Spiller GA, Jensen CD, Ellis BK Gates JE. Role of water-soluble dietary fiber in the management of elevated plasma cholesterol in healthy subjects. Am $\mathcal{F}$ Cardiol 1992;69:433- 